\title{
Path Stretching and Tracking for Time-Based Aircraft Spacing at Meter Fix
}

\author{
Thierry Miquel $^{*}$ \\ Direction des Services de la Navigation Aérienne (Etudes et Recherche appliquée), Toulouse, FRANCE \\ Félix Mora-Camino ${ }^{\dagger}$ \\ Ecole Nationale de l'Aviation Civile and LAAS du CNRS, Toulouse, FRANCE \\ and \\ Jean-Marc Loscos* \\ Direction des Services de la Navigation Aérienne (Etudes et Recherche appliquée), Toulouse, FRANCE
}

\begin{abstract}
The delegation to the flight crew of some tasks currently performed by air traffic controllers provides new perspectives potentially to increase air traffic control efficiency. More specifically, the task of establishing properly spaced landing sequences is very demanding in heavy traffic conditions for the air traffic controllers in charge of the terminal maneuvering area. Automatic merging operations could relieve air traffic controllers of providing time-consuming radar-vectoring instructions and could provide more optimal flight profiles to the aircraft. With these new tasks being transferred to the flight crew some automation in order to limit the workload is required. The outcomes of this paper are twofold: firstly it presents a technique to generate path stretching with length and endpoint constraints and secondly it provides a nonlinear control law to track the reference trajectory. The reference trajectory is used to stretch the path of a trailing aircraft in order to delay its arrival on a meter fix. The purpose of the nonlinear control law based on feedback linearization is to enable the trailing aircraft to follow the reference trajectory. This communication treats level flight, but wind is considered. The design is followed by two illustrative examples which show the effectiveness of the proposed approach.
\end{abstract}

\section{Introduction}

$\mathrm{T}$ he overall goal of the R\&D community has always been to improve Air Traffic Management (ATM) operations through a greater involvement of pilots in cooperation with air traffic controllers. The technology called ADS-B (Automatic Dependent Surveillance-Broadcast) is now available for civil pilots/aircraft ${ }^{1}$ to enhance the rudimentary Flight Information Services (FIS) today relayed to them by air traffic controllers. In addition, the Airborne Separation Assistance System (ASAS) concept was proposed to ICAO in March $1995^{2}$ to take advantage of the airborne capabilities in particular for communication, navigation and surveillance. The range of ASAS applications is very wide ${ }^{3}$, from the use of flight deck traffic displays enhancing visual operations to more autonomous aircraft operations. There is no "ASAS concept" per se; ASAS and its operational applications are only components of the overall ATM system. ASAS applications are designed to be fully integrated into ATM operations, and most of them involve the active cooperation of air traffic controllers and pilots.

ASAS incorporates an airborne surveillance function, meaning that the flight crew has an electronic display of the surrounding airspace. In practice, ADS-B is the technical support system for ASAS. ASAS will use the airborne

\footnotetext{
* PhD, Civil Aviation Design and Operation Engineer, Airborne Surveillance, Collision Avoidance and Separation Division,miquel@cena.fr

${ }^{\dagger}$ Senior Researcher at LAAS du CNRS, Professor of Automatic Control and Avionics at Ecole Nationale de l'Aviation civile, Felix.MORA@enac.fr

* Civil Aviation Design and Operation Engineer, Head of Airborne Surveillance, Collision Avoidance and Separation Division, loscos@cena.fr Copyright $\odot 2006$ by DSNA. Published by the American Institute of Aeronautics and Astronautics, Inc., with permission.
} 
surveillance function (called ADS-B IN), but will also need an ASAS logic and a control panel (HMI) for the flight crew.

By ICAO definition ${ }^{4}$ ASAS is an airborne system that allows the flight crew to maintain separation between their aircraft and one or more other aircraft, and provides information concerning the surrounding traffic. ASAS on board an aircraft interacts with the existing systems (particularly the FMS) or even with the installed equipment (particularly the display screens); this may also require new equipment depending on the architecture needed. Not only the system, but also its utilization was defined by ICAO: an ASAS application is a set of operational procedures for air traffic controllers and flight crews, which makes use of the capabilities of airborne separation assistance systems to meet a clearly-defined operational objective.

Basic expectations about ASAS concern safety, capacity and reduction in cost. Indeed, situational awareness would be reinforced in the flight deck, with the possibility of a better understanding of the traffic in the vicinity. It would complement visual acquisition and avoid visual acquisition errors. It would not replace the safety nets provided by short term conflict alerts (STCA) or Airborne Collision Avoidance System (ACAS), but would help to avoid situations involving the safety nets by reducing the exposure to errors of judgement in planning the air traffic situation. In addition, the ASAS applications should lead to a reduction in the controller's workload. The flight crew workload will be related to the automation associated with the ASAS. According to the air traffic conditions, the specific airspace constraints, and the controller's choices, this reduction in workload could result in an increase in capacity and/or efficiency of flights. These gains depend on many factors. For example the situational awareness provided to the pilot by ASAS could avoid requests to traffic information which in turn would contribute to a reduction in the controller's workload. Finally, the cost reduction could come from the reduction in delays associated with gains in capacity and efficiency. A reduction in costs linked to the ATC infrastructure is not expected in the implementation time considered.

This paper considers "Airborne Spacing" applications. In this category of ASAS applications for maintaining spacing (without transfer of responsibility) the flight crews must acquire and maintain a given distance or time with another designated aircraft, after a new ATC instruction. Even if the flight crews inherit new tasks, the separation between aircraft is still the controller's responsibility. This ASAS application can thus be regarded as a new instruction based on the aircraft's new capabilities, on the controller's initiative. Amongst the various applications considered, "Sequencing \& Merging" appears to be promising for sequencing aircraft that arrive at large terminal maneuvering areas (TMA).

The task of establishing properly spaced landing sequences is very demanding in heavy traffic conditions for the air traffic controllers in charge of the terminal maneuvering area. Automatic merging operations could relieve air traffic controllers of the need to provide time-consuming radar-vectoring instructions. On the airborne side, this would provide means to anticipate the changes to the trajectory as well as the speed reductions.

This paper focuses on path stretching and tracking for time-Based aircraft spacing at meter fix. The path stretching problem consists in finding curves that satisfy constraints on the positions and tangents at the two endpoints. Finding such curves is nontrivial. Indeed, splines ${ }^{5}$ or clothoid ${ }^{6}$ curves tend to produce smooth path with continuous curvature that can match arbitrary endpoint constraints, but the length of the achieved trajectories are variable. Other works focus on finite-element methods where the curve may be represented by a large number of line segments which preserve the length ${ }^{7}$ but make planning rather difficult.

The outcomes of this paper are twofold: firstly a technique to generate path stretching with length and endpoint constraints is presented, and secondly a nonlinear control law to track the reference trajectory is proposed. The reference trajectory is used to stretch the path of a trailing aircraft in order to delay its arrival on a meter fix. The purpose of the nonlinear control law based on feedback linearization is to enable the trailing aircraft to follow the reference trajectory.

The paper is organized as follows: in the preliminaries, the envisioned functional architecture onboard aircraft and kinematics equations are presented. Then the proposed path-stretching technique is presented. Curves of constant length that satisfy constraints on the position and tangents at the two endpoints are considered. The subsequent section presents the control of the lateral motion of the trailing aircraft. Two illustrative examples are then presented in order to show the effectiveness of the proposed approach. Finally, some conclusions are put forward. 


\section{Preliminaries}

\section{A. Envisioned functional architecture}

In the following, a fully coordinated aircraft (i.e. side-slip angle is assumed to be zero) flying over a flat and nonrotating earth within standard atmosphere is assumed. Furthermore, and as in many modern jets, it is assumed in the following that a Flight Management System (FMS) and an autopilot function dealing with heading control is available onboard the trailing aircraft.

Insofar as speed, altitude and time constraints are generally collocated with a way-point ( 2 dimensional point) within $\mathrm{FMS}^{8}$, the path stretching feedback loop is envisioned as part of new FMS functionalities, as depicted in Figure 1:

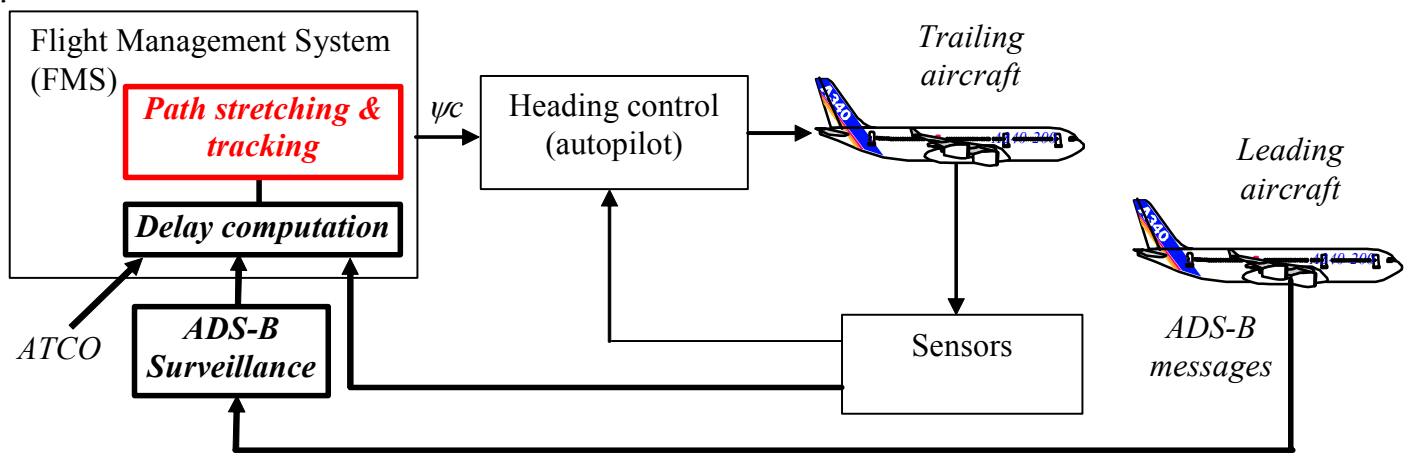

Figure 1: Path stretching \& tracking feedback loop

Three functionalities would need to be added to current FMS in order to achieve time-based aircraft relative guidance, prefiguring future ASAS equipment embedded in FMS:

- ADS-B surveillance: the purpose of this function is to track the position of the leading aircraft;

- Delay computation: the purpose of this function is to estimate the delay between the leading aircraft and the trailing aircraft at a given meter fix assigned by the air traffic controller.

- Path stretching: the purpose of this function is to compute a new trajectory with length and endpoint constraints.

This paper focuses on the path-stretching aspect at constant airspeed. Furthermore, this paper is restricted to the case where the flight is level, but wind is considered and consequently the groundspeed may be variable.

\section{B. Kinematics equations}

The kinematics equations of motion of the trailing aircraft are (see Figure 2):

$$
\left\{\begin{array}{l}
\dot{x}=V \cos (\psi)-W \cos \left(\psi_{w}\right)=G s \cos (\chi) \\
\dot{y}=V \sin (\psi)-W \sin \left(\psi_{w}\right)=G s \sin (\chi)
\end{array}\right.
$$

Where $\psi_{w}$ is the direction from where the wind is blowing, $W$ is the speed of the wind, $\psi$ is the heading of the aircraft and $V$ its airspeed, $G s$ is groundspeed and $\chi$ the ground track.

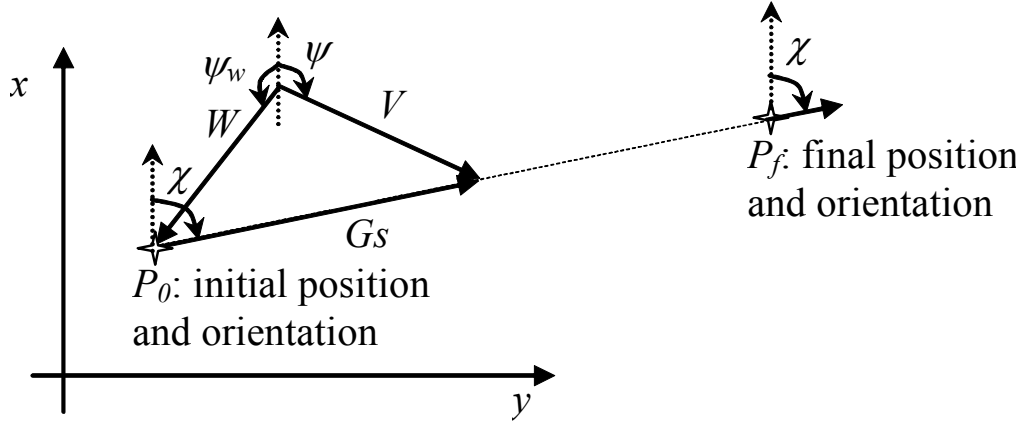

Figure 2: Position, orientation and ground speed computation

Following Figure 2, the ground speed $G s$ is given by:

$$
\vec{V}=\overrightarrow{G s}+\vec{W} \Rightarrow G s^{2}+2 \cdot G s \cdot W \cdot \cos \left(\chi-\psi_{w}\right)+W^{2}-V^{2}=0
$$


Solving this quadratic equation in $G s$ and assuming that the angle difference between the heading $\psi$ and the ground track $\chi$ is less than 90 degrees leads to:

$$
G s=-W \cos \left(\chi-\psi_{w}\right)+\sqrt{V^{2}-W^{2} \sin ^{2}\left(\chi-\psi_{w}\right)} \text { where }-W \cos \left(\chi-\psi_{w}\right) \geq 0
$$

\section{Path stretching}

\section{A. Parameterization}

The objective for the path planning is to stretch the trajectory while maintaining a smooth motion. The problem consists of generating a smooth trajectory curve by joining two distinct configurations (the oriented start and end points) with constraints on time of arrival.

In this paper, the continuous curvature on the trajectory is based on a sinusoidal parameterization ${ }^{9}$ of the heading angle $\psi(t)$ of the aircraft:

$$
\psi(t)=\psi(0)+a \cdot\left(\sin \left(2 \pi \cdot \frac{t}{T}-\delta\right)+\sin (\delta)\right)
$$

Where $\psi(0)$ is the heading of the aircraft at the beginning of the maneuver and $T$ is the time needed by the aircraft to achieve the maneuver. This time is provided by the delay computation functionality, and is the sum of the estimated time of arrival of the leading aircraft on the meter fix and the delay between the two aircraft required by the air traffic controller.

Integration of kinematics equation (1) uses the following property of Bessel functions ${ }^{12}$ :

$$
J_{n}(x)=\frac{1}{\pi} \int_{0}^{\pi} \cos (n \cdot y-x \cdot \sin (y)) \cdot d y=\int_{0}^{1} \cos (n \cdot y-x \cdot \sin (\pi y)) \cdot d y
$$

Parameterization (4) of the heading angle leads to the following expression of the position of the trailing aircraft at time $T$ :

$$
\left\{\begin{array}{l}
x(T)=x(0)+T \cdot\left(V \cdot J_{0}(a) \cdot \cos (\psi(0)+a \cdot \sin (\delta))-W \cdot \cos \left(\psi_{w}\right)\right) \\
y(T)=y(0)+T \cdot\left(V \cdot J_{0}(a) \cdot \sin (\psi(0)+a \cdot \sin (\delta))-W \cdot \sin \left(\psi_{w}\right)\right)
\end{array}\right.
$$

Here $J_{0}(a)$ is the zero-order Bessel function of the first kind.

Parameter $a$ is obtained from (6) by solving the following equations (see Figure 2):

$$
\begin{aligned}
& \left\{\begin{array}{l}
x(T)-x(0)+W \cdot T \cdot \cos \left(\psi_{w}\right)=V \cdot T \cdot J_{0}(a) \cdot \cos (\psi(0)+a \cdot \sin (\delta)) \\
y(T)-y(0)+W \cdot T \cdot \sin \left(\psi_{w}\right)=V \cdot T \cdot J_{0}(a) \cdot \sin (\psi(0)+a \cdot \sin (\delta))
\end{array}\right. \\
& \Rightarrow\left\{\begin{array}{l}
J_{0}(a)=\frac{\sqrt{d_{T}^{2}+(W \cdot T)^{2}+2 \cdot W \cdot T \cdot d_{T} \cdot \cos \left(\chi-\psi_{w}\right)}}{V \cdot T} \\
\text { where } d_{T}=\sqrt{(x(T)-x(0))^{2}+(y(T)-y(0))^{2}}
\end{array}\right.
\end{aligned}
$$

At time $t=T$ and when the wind speed $W$ is null (that is $\delta=0$ ), the situation is similar to the situation where the trailing aircraft flies at constant heading $\psi(0)$, but with an airspeed reduced to $V \cdot J_{0}(a)$ rather than $V$.

At point $P_{f}$ the track must be $\chi$, so using (1) and (6) we have:

$$
\frac{y(T)-y(0)}{x(T)-x(0)}=\tan (\chi) \Leftrightarrow \frac{V \cdot J_{0}(a) \cdot \sin (\psi(0)+a \cdot \sin (\delta))-W \cdot \sin \left(\psi_{w}\right)}{V \cdot J_{0}(a) \cdot \cos (\psi(0)+a \cdot \sin (\delta))-W \cdot \cos \left(\psi_{w}\right)}=\tan (\chi)
$$

Let us define angle $\theta$ by:

Thus, we get when developing (8):

$$
\theta=\psi(0)+a \cdot \sin (\delta)
$$

$$
\sin (\theta) \cdot \cos (\chi)-\cos (\theta) \cdot \sin (\chi)=\sin (\theta-\chi)=\frac{W}{V \cdot J_{0}(a)} \cdot\left(\sin \left(\psi_{w}\right) \cdot \cos (\chi)-\cos \left(\psi_{w}\right) \cdot \sin (\chi)\right)
$$

So, we finally get:

$$
\delta=\arcsin \left(\frac{\theta-\psi(0)}{a}\right) \text { where } \theta=\chi+\arcsin \left(\frac{W}{V \cdot J_{0}(a)} \cdot\left(\sin \left(\psi_{w}\right) \cdot \cos (\chi)-\cos \left(\psi_{w}\right) \cdot \sin (\chi)\right)\right)
$$




\section{B. Dynamics of the reference trajectory}

The dynamics of the reference trajectory are derived from (1) and (4):

$$
\begin{aligned}
& \left\{\begin{array}{l}
\dot{x}_{d}(t)=V \cos \left(\psi_{d}(t)\right)-W \cos \left(\psi_{w}\right) \\
\dot{y}_{d}(t)=V \sin \left(\psi_{d}(t)\right)-W \sin \left(\psi_{w}\right) \\
\dot{\psi}_{d}(t)=r_{d} \\
\dot{r}_{d}(t)=-\left(\frac{2 \pi}{T}\right)^{2} \cdot\left(\psi_{d}(t)-\psi(0)-a \cdot \sin (\delta)\right)
\end{array}\right. \\
& \text { Where } \psi_{d}(t)=\psi(0)+a \cdot\left(\sin \left(2 \pi \cdot \frac{t}{T}-\delta\right)+\sin (\delta)\right)
\end{aligned}
$$

The initial conditions are:

$$
\left\{\begin{array}{l}
x_{d}(0)=x(0) \\
y_{d}(0)=y(0) \\
\psi_{d}(0)=\psi(0) \\
r_{d}(0)=\frac{2 \pi \cdot a \cdot \cos (\delta)}{T}
\end{array}\right.
$$

\section{Path Tracking}

The purpose of path tracking is to make the aircraft able to follow the reference trajectory established by the path stretching algorithm.

The path tracking control the lateral motion of the trailing aircraft by providing heading cues to the autopilot. It is based on feedback linearization ${ }^{10}$ which allows the transformation of a nonlinear system into an equivalent linear system. From Figure 3, the cross track distance $v$ and the along track distance $\tau$ from the current position $(x, y)$ of the trailing aircraft to the axis oriented by the desired track $\chi_{d}$ and passing through the desired position $\left(x_{d}, y_{d}\right)$ are given by:

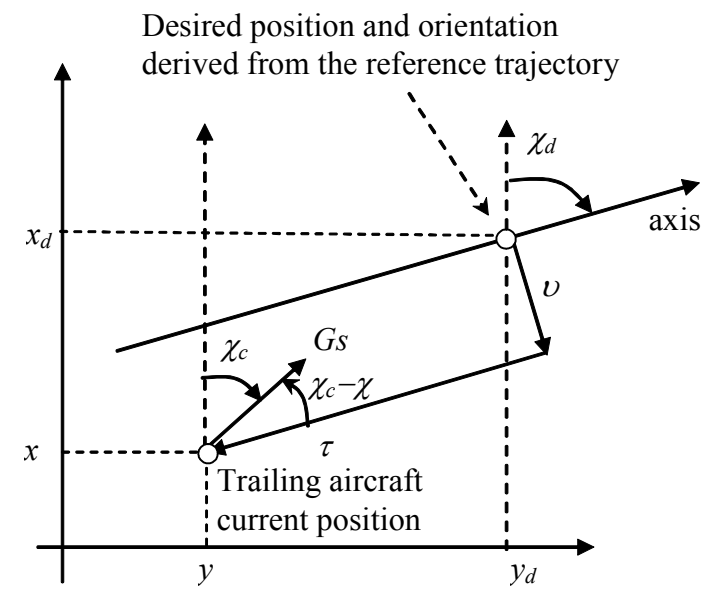

Figure 3: Cross track distance $v$ and the along track distance $\tau$

$$
\left[\begin{array}{l}
\tau \\
v
\end{array}\right]=\left[\begin{array}{cc}
\cos \left(\chi_{d}\right) & \sin \left(\chi_{d}\right) \\
-\sin \left(\chi_{d}\right) & \cos \left(\chi_{d}\right)
\end{array}\right] \cdot\left[\begin{array}{c}
x-x_{d} \\
y-y_{d}
\end{array}\right]
$$

In addition, the dynamics of the cross track distance $v$ is given by:

$$
\frac{d}{d t} v=G s \cdot \sin \left(\chi_{c}-\chi_{d}\right)
$$

Now, let us impose a first order dynamic for the cross track distance:

$$
\frac{d}{d t} v=-\lambda \cdot v
$$

Combining the two previous equations leads to the expression for the commanded track angle $\chi_{c}$ : 


$$
-\lambda \cdot v=G s \cdot \sin \left(\chi_{c}-\chi_{d}\right) \Leftrightarrow \chi_{c}=\chi_{d}-\arcsin \left(\frac{\lambda \cdot v}{G s}\right)
$$

Previous equation shows that the ratio $\lambda \cdot v / G s$ shall lie between -1 and +1 . In addition, the difference between the desired track $\chi_{d}$ and the commanded track $\chi_{c}$ shall be limited between $-\pi / 2$ and $+\pi / 2$.

The previous equation associated with (1) leads to the following expression for the commanded heading angle $\psi_{c}$ :

$$
\left\{\begin{array}{l}
\psi_{c}=\arctan \left(\frac{G s \cdot \sin \left(\chi_{c}\right)+W \cdot \sin \left(\psi_{w}\right)}{G s \cdot \cos \left(\chi_{c}\right)+W \cdot \cos \left(\psi_{w}\right)}\right)=\arctan \left(\frac{G s \cdot \sin \left(\chi_{d}-\arcsin \left(\frac{\lambda \cdot v}{G s}\right)\right)+W \cdot \sin \left(\psi_{w}\right)}{G s \cdot \cos \left(\chi_{d}-\arcsin \left(\frac{\lambda \cdot v}{G s}\right)\right)+W \cdot \cos \left(\psi_{w}\right)}\right) \\
G s=\sqrt{V^{2}+W^{2}-2 \cdot V \cdot W \cdot \cos \left(\psi_{d}-\psi_{w}\right)}
\end{array}\right.
$$

In the following, the ratio between the airspeed $V$ and parameter $\lambda$ has been set to the radius of turn $R$ of the aircraft:

$$
\frac{V}{\lambda}=R=\frac{V^{2}}{g \cdot \tan \left(\varphi_{\max }\right)} \Rightarrow \lambda=\frac{g \cdot \tan \left(\varphi_{\max }\right)}{V}
$$

Assuming a maximum bank angle $\varphi_{\max }$ of 30 degrees and an airspeed $V$ of $149 \mathrm{~m} / \mathrm{s}$, this leads to a value of parameter $\lambda$ at $0.038 \mathrm{sec}^{-1}$.

\section{Illustrative examples}

\section{A. Scenarios}

In this section, two situations are presented in order to evaluate the properties of the path stretching and tracking control previously described.

The aircraft model which has been used is a fictitious commercial aircraft model (called RCAM) which was developed by the Group for Aeronautical Research and Technology in Europe ${ }^{11}$ (GARTEUR). The aircraft mass has been set to $120000 \mathrm{~kg}$. The airspeed (TAS) of the aircraft has been chosen to be $289 \mathrm{knots}(149 \mathrm{~m} / \mathrm{s}) \mathrm{which}$ corresponds to a conventional airspeed (CAS) of 250 knots at flight level 100. This airspeed is compliant with current procedure and observations on radar data.

The scenario is depicted in Figure 4, where the aircraft is supposed to go from meter fix DPE (Dieppe) to SOKMU, which are $37 \mathrm{NM}$ separated. This is built around real life meter fixes of an air navigation procedure in France.

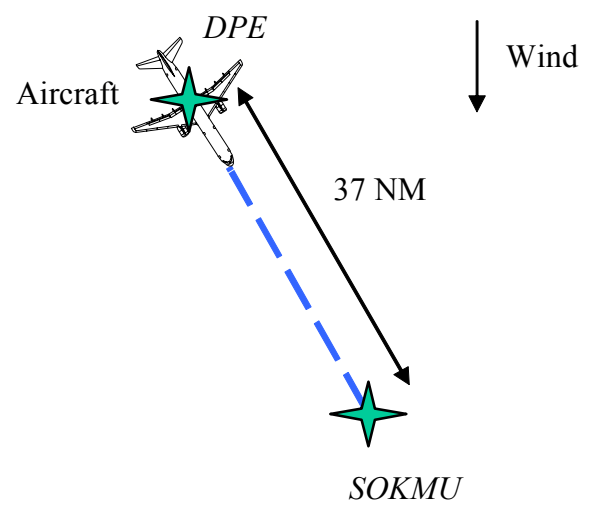

Figure 4: Case study scenario

In the first situation, no wind is present and the aircraft reaches SOKMU at $\mathrm{t}=460 \mathrm{sec}$, which is the time needed to fly $37 \mathrm{NM}$ at an airspeed of 289 knots.

In the second situation, a wind of 40 knots $(20 \mathrm{~m} / \mathrm{s})$ blowing from the North is considered. The aircraft reaches SOKMU at $\mathrm{t}=408 \mathrm{sec}$, which is the time needed to fly $37 \mathrm{NM}$ at a groundspeed of $326 \mathrm{knots}(168 \mathrm{~m} / \mathrm{s})$.

The objective of the simulation is to delay the arrival time of the aircraft to SOKMU by 90 sec for both situations. So, the time $T$ used in (7) is respectively $460+90 \mathrm{sec}$ for the situation with no wind, and $408+90 \mathrm{sec}$ for the situation with wind. The value of distance $d_{T}$ used in (7) is $37 \mathrm{NM}$. 


\section{B. Results when no wind is considered}

For the first situation, when there is no wind, the parameter $a$ of the Bessel function and the angle $\delta$ have the following values:

$$
\left\{\begin{array}{l}
a=0.8266 \\
\delta=0
\end{array}\right.
$$

The arrival time of the aircraft to SOKMU is $552 \mathrm{sec}$ that is to say that the trajectory has been delayed by $92 \mathrm{sec}$ (552-460). The time difference of $2 \mathrm{sec}$ between the desired delay of $90 \mathrm{sec}$ and the achieved delay of $92 \mathrm{sec}$ is due to the path tracking.

The trajectory and the controlled heading of the aircraft for the this situation is presented in Figures 6:

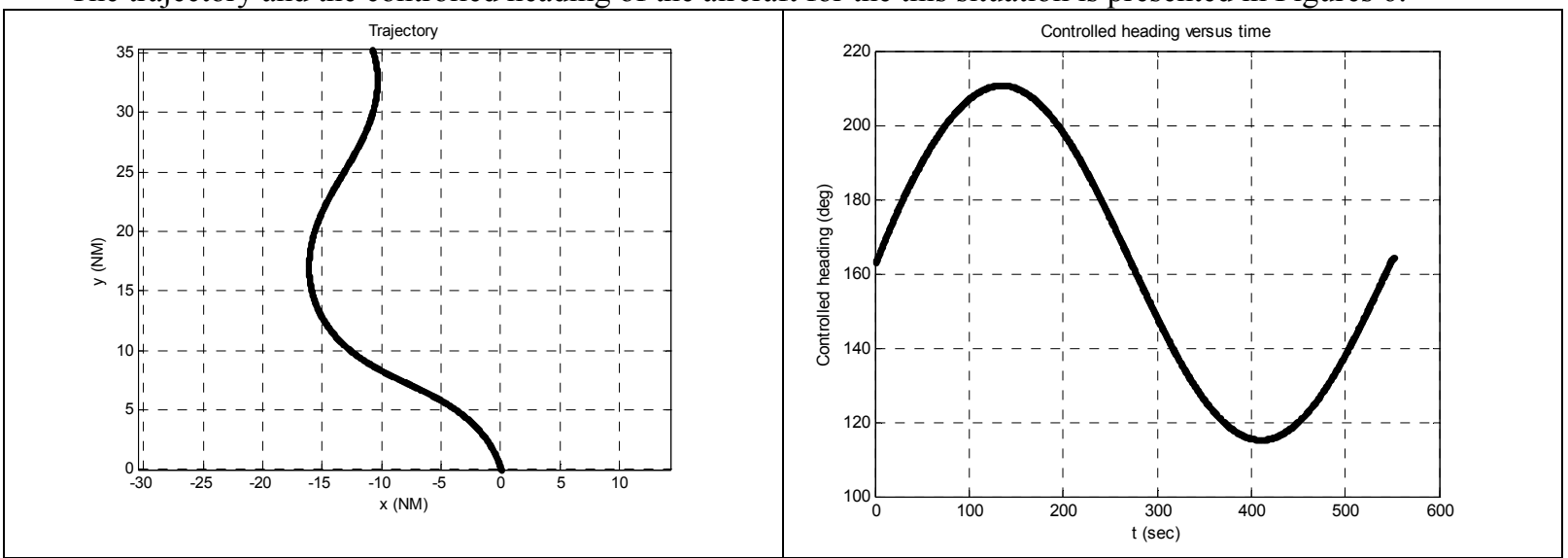

Figure 6: Trajectory and the controlled heading for the no wind situation

\section{Results when wind is considered}

For the second situation, when the wind is blowing 40 knots from North, the parameter $a$ of the Bessel function and the angle $\delta$ have the following values:

$$
\left\{\begin{array}{l}
a=0.9272 \\
\delta=-0.0108
\end{array}\right.
$$

The arrival time of the aircraft to SOKMU is $500 \mathrm{sec}$ that is to say that the trajectory has been delayed by $92 \mathrm{sec}$ (500-408).

The trajectory and the controlled heading of the aircraft for the this situation is presented in Figures 7 :

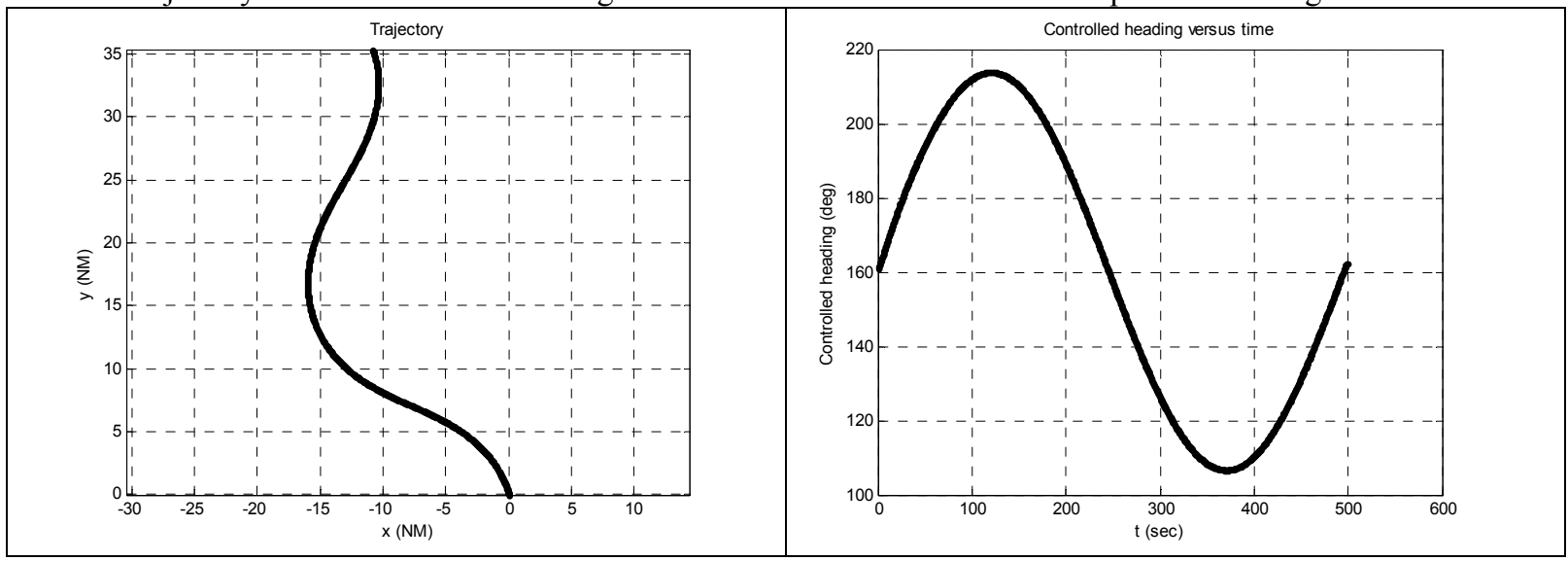

Figure 7: Trajectory and the controlled heading for the wind situation

Despite the presence of wind, the trajectory is very similar to that one achieved when no wind is considered. This is due to differences in the controlled heading. 


\section{Conclusion}

In this paper, the design of a new FMS function dedicated to path stretching has been considered. This new function aims at achieving a specified delay between commercial aircraft at a specified meter fix.

This envisioned new capability onboard commercial aircraft takes advantage of the Automatic Dependent Surveillance-Broadcast $(A D S-B)$ and provides new perspectives to potentially increase air traffic control efficiency. It could be seen as the airborne counterpart of the ground based arrival manager.

In this paper, the path stretching technique is based on a sinusoidal parameterization of the heading angle, whereas the tracking of the reference trajectory is made through heading control which is based on feedback linearization. This allows the trailing aircraft to accurately follow the reference trajectory. Airspeed is maintained at a constant value in order to avoid throttle demand

Simulation results illustrate the efficiency of the proposed design. Nevertheless, additional studies in terms of operational scenarios, which take into account the vertical motion of the aircraft, are needed in order to refine and validate the proposed design. Future work will focus on the assessment of the proposed design using a set of numerous simulated encounters for the purpose of statistical evaluation. In addition adaptive path stretching will be addressed in order to take into account possible airspeed variations.

\section{Acknowledgments}

The authors wish to thank Philippe Louyot for helpful inputs and comments.

\section{References}

1 The Eleventh ICAO Air Navigation Conference, Automatic dependent surveillance - broadcast (Concept of Use), WP6 Item 1.2 in the list of documentation AN-Conf11 (2003)

(http://www.icao.int/icao/en/anb/meetings/anconfl1/index.html)

2 Air Traffic Control Quarterly, Volume 13 Number 2 Special Issue on ASAS, Francis Casaux Editor (2005)

3 Loscos J.M., ASAS: towards new cooperation based on airborne separation, Revue technique de la DTI no. 2, pp. 69-82 (2005)

4 The Eleventh ICAO Air Navigation Conference, Circular on airborne separation assistance system (ASAS), IP5 Item 1.2 in the list of documentation AN-Confl1 (2003)

(http://www.icao.int/icao/en/anb/meetings/anconfl 1/index.html)

5 Yamamo M., Iwamura M., Mohri A., Quasic-Time-Optimal Motion Planning of Mobile Platforms in the Presence of Obstacles, International Conference on Robotics and Automation, pp. $739-744$ (1999)

6 Fraichard Th., Ahuactzin J. M., Smooth Path Planning for Cars, IEEE International Conference On Robotics and Automation May, pp. 21-26 (2001)

7 Lamiraux F., Kavraki L. E., Planning paths for elastic objects under manipulation constraints, International Journal of Robotics Research, vol. 20, no. 3, pp. 188-208 (2001)

8 Hoffman E., Levrez J.M., Flight Management and Guidance Control System Model for an ATC Simulation Traffic Generator, EEC REPORT NO. 303 (1996)

$9 \quad$ Moll M., Kavraki L. E., Path planning for minimal energy curves of constant length, IEEE Conference on Robotics and Automation, pp. 2826-2831 (2004)

10 Khalil H.K., Nonlinear Systems, Prentice Hall, Third Edition (2002)

11 Magni J.F., Bennani S., Terlouw J., Robust Flight Control : A Design Challenge, Springer-Verlag Publications (1997)

12 Bowman F., Introduction to Bessel Functions, Dover Publications (1958) 